Arq. Bras. Med. Vet. Zootec., v.67, n.3, p.945-949, 2015

\title{
Comunicação
}

[Communication]

\section{Aloe vera na criopreservação do sêmen de tambaqui (Colossoma macropomum)}

\author{
[Aloe vera in the cryopreservation of tambaqui (Colossoma macropomum) sperm]
}

M.A.P. Melo-Maciel, L.V. Leite-Castro, J.S. Leite, M.S. Oliveira, P.S. Almeida-Monteiro, J.F. Nunes, C.S.B. Salmito-Vanderley

Aluna de pós-graduação - Universidade Estadual do Ceará - Fortaleza, CE

O Colossoma macropomum, tambaqui, é uma espécie de peixe migrador pertencente à ordem Characiforme, oriunda da bacia Amazônica e do rio Orinoco, sendo a espécie nativa mais produzida em cativeiro (Streit Jr. et al., 2012).

Estudos de criopreservação de sêmen utilizando a Água de Coco em Pó (ACP) já foram testados com sucesso em diferentes espécies de peixes, como o Prochilodus lineatus (Viveiros et al., 2010), Colossoma macropomum (Leite et al., 2011) e Piaractus brachypomus (Melo, 2010b), assim como o crioprotetor interno Dimetilsufóxido (DMSO) (Salmito-Vanderley et $a l .$, 2012). No entanto, sua associação com outro produto de origem vegetal, como o extrato bruto de Aloe vera, ainda não foi testada. Tal extrato, por possuir composição complexa (rico em açúcares, minerais, entre outros) (Veloso e Peglow, 2003), pode fornecer nutrição e proteção às células espermáticas, podendo atuar como crioprotetor externo.

Dessa forma, por não haver relatos sobre a utilização de Aloe vera (AV) na conservação de sêmen de peixes, o objetivo deste trabalho foi testar o extrato bruto de AV em diferentes concentrações, associado ou não ao DMSO, na criopreservação do sêmen de tambaqui.

Este trabalho foi aprovado pelo Comitê de Ética para Uso Animal (CEUA) da Universidade Estadual do Ceará com protocolo $\mathrm{n}^{\circ} 11518754$ $5 / 74$.

Recebido em 2 de julho de 2014

Aceito em 3 de março de 2015

E-mail: monicaaline3@hotmail.com
Durante a estação reprodutiva, foram utilizados 30 machos de tambaqui (C. macropomum) provenientes do plantel do Departamento Nacional de Obras Contra as Secas (DNOCS) em Pentecoste-CE. Os animais foram utilizados duas vezes durante o experimento, sendo pesados, medidos e induzidos hormonalmente com uma dose de extrato de hipófise de carpa de $2 \mathrm{mg} / \mathrm{kg}$ de peso vivo (Leite et al., 2011; Carneiro et al., 2012), 14 horas antes da coleta de sêmen.

Na primeira coleta de sêmen, 24 animais tiveram sêmen válido para sua utilização no experimento, formando 8 pools. Na segunda coleta, apenas 18 animais tiveram amostras válidas, gerando 6 pools. Totalizaram-se assim 14 pools (três animais para cada pool). As amostras válidas (Leite et al., 2011) tiveram pH, volume e concentração espermática mensurados.

Cada pool foi aliquotado para criopreservação e osmolaridade. Além disso, análise da morfologia espermática e análise computadorizada da motilidade espermática com auxílio do Sperm Class Analyser (SCA; Microptics, Espanha) foram realizadas antes da criopreservação e após a descongelação Nessa análise computadorizada, os pools foram ativados com $\mathrm{NaCl}$ a $50 \mathrm{mM}$ $(1: 50 ;$ sêmen: $\mathrm{NaCl})$ e observados quanto à motilidade total, velocidade curvilinear (VCL), velocidade média do percurso (VAP) e velocidade em linha reta (VSL).

A análise da morfologia espermática foi realizada a partir da fixação de cada pool em formol-citrato $4 \% \quad(1: 10 ;$ sêmen:fixador $) \quad \mathrm{e}$ 
coloração com Rosa Bengala (3:20; corante:sêmen fixado). Foram observados 200 espermatozoides (de cada pool, em cada tratamento) em microscópio de luz (400x), e avaliada a taxa de espermatozoides morfologicamente normais.

A osmolaridade dos pools foi mensurada por meio da utilização de $100 \mu \mathrm{L}$ da amostra em osmômetro digital de refrigeração Peltier (Roebling, Alemanhã).

Para a criopreservação, o ACP-104 (Água de Coco em Pó, diluidor seminal, ACPBiotecnologia, Brasil) foi preparado conforme recomendações do fabricante e utilizado em todos os tratamentos na mesma proporção. Para a utilização do extrato bruto de Aloe vera (AV), seguiu-se o método utilizado por Melo (2010a).

Os pools foram diluídos em meio à base de ACP104, na proporção de 1:6 (sêmen:diluidor), adicionando diferentes crioprotetores, de maneira a constituir 5 tratamentos, a saber: T1: $10 \%$ DMSO; T2: $10 \%$ DMSO + 5\% AV; T3: $10 \%$ DMSO + 10\% AV; T4: 5\% AV; T5: 10\% AV.

A criopreservação foi realizada seguindo o protocolo de Leite et al. (2011). As palhetas foram armazenadas em botijão criogênico. A descongelação foi realizada 15 dias após a criopreservação, removendo as palhetas do botijão e imergindo-as em banho-maria a $40^{\circ} \mathrm{C}$ por 20 s.

Os resultados foram apresentados na forma de média \pm desvio padrão. As variáveis estudadas (motilidade total, VCL, VSL, VAP, taxa de espermatozoides morfologicamente normais) foram analisadas para normalidade pelo teste de Shapiro-Wilk, e para homocedasticidade, pelo teste de Levene. Variáveis percentuais que não apresentaram distribuição normal mesmo após transformação angular (arcsen $\sqrt{ }$ ) foram consideradas como não paramétricas. Apenas a variável taxa de espermatozoides morfologicamente normais apresentou distribuição normal, e as diferenças entre os tratamentos para essa variável foram avaliadas pela Análise de Variância (ANOVA), seguida de comparação entre as médias pelo teste de Student-Newman-Keuls (SNK) ao nível de significância de 5\%. As variáveis não paramétricas foram comparadas pelo teste de Kruskal-Wallis, seguido pelo teste de comparação múltipla de Dunn a $5 \%$ de significância. Os coeficientes de correlação entre as variáveis estudadas (motilidade total, VCL, VSL, VAP, taxa de espermatozoides morfologicamente normais) foram estimados pelo teste de correlação de postos de Spearman. As análises estatísticas foram realizadas no software estatístico $\quad$ SPSS $\quad\left(\right.$ IBM $^{\circledR} \quad$ SPSS $^{\circledR}$ Statistics versão 20).

As médias obtidas do volume seminal coletado foi de $2,84 \pm 2,15 \mathrm{~mL}$, com $\mathrm{pH}$ variando entre 8 e 9, motilidade subjetiva $89,31 \pm 4,79 \%$, concentração espermática por $\mathrm{mL}$ de $25,22 \pm 9,81 \times 10^{9}$. O peso e comprimento dos animais foram $5,11 \pm 1,29 \mathrm{~kg}$ e $62,95 \pm 5,66 \mathrm{~cm}$, respectivamente. A média da osmolaridade dos pools foi de $292,84 \pm 15,74 \mathrm{mOsm} . \mathrm{kg}^{-1}$.

Tabela 1. Motilidade e velocidades espermáticas do sêmen de tambaqui criopreservado em ACP-104 adicionado de diferentes crioprotetores (média \pm desvio padrão)

\begin{tabular}{lcccc}
\multicolumn{1}{c}{ Tratamentos } & $\begin{array}{c}\text { Motilidade } \\
\text { total }(\%)\end{array}$ & $\begin{array}{c}\text { VSL } \\
\mu \mathrm{m}^{-1}\end{array}$ & $\begin{array}{c}\text { VAP } \\
\mu \mathrm{m} \cdot \mathrm{s}^{-1}\end{array}$ & $\begin{array}{c}\text { VCL } \\
\mu \mathrm{m} \cdot \mathrm{s}^{-1}\end{array}$ \\
\hline Sêmen fresco & $94,36 \pm 5,02 \mathrm{a}$ & $33,57 \pm 16,87 \mathrm{a}$ & $56,93 \pm 16,87 \mathrm{a}$ & $73,36 \pm 35,17 \mathrm{a}$ \\
T1: $10 \%$ DMSO & $20,86 \pm 8,31 \mathrm{~b}$ & $19,71 \pm 5,92 \mathrm{~b}$ & $27,79 \pm 5,51 \mathrm{~b}$ & $36,21 \pm 6,19 \mathrm{~b}$ \\
T2: $10 \%$ DMSO+5\%AV & $15,71 \pm 9,77 \mathrm{~b}, \mathrm{c}$ & $15,93 \pm 5,97 \mathrm{~b}, \mathrm{c}$ & $24,14 \pm 4,13 \mathrm{~b}$ & $32,79 \pm 3,79 \mathrm{c}$ \\
T3: $10 \%$ DMSO+10\%AV & $10,57 \pm 3,94 \mathrm{c}$ & $12,86 \pm 2,29 \mathrm{c}$ & $20,36 \pm 2,82 \mathrm{c}$ & $29,64 \pm 6,73 \mathrm{c}$ \\
T4: $5 \%$ AV & $7,64 \pm 2,13 \mathrm{~d}$ & $17,86 \pm 6,01 \mathrm{~b}, \mathrm{c}$ & $27,21 \pm 4,85 \mathrm{~b}$ & $38,64 \pm 6,44 \mathrm{~b}$ \\
T5: $10 \%$ AV & $7,00 \pm 2,08 \mathrm{~d}$ & $17,23 \pm 4,90 \mathrm{~b}, \mathrm{c}$ & $27,15 \pm 3,51 \mathrm{~b}$ & $37,38 \pm 4,96 \mathrm{~b}$ \\
\hline
\end{tabular}

Letras diferentes na coluna diferem significativamente entre tratamentos. $\mathrm{P}<0,05$.

A motilidade total dos espermatozoides e as velocidades espermáticas após a criopreservação apresentaram uma redução significativa quando comparadas às do sêmen fresco (Tab. 1). Em relação aos tratamentos, a motilidade total apresentou melhores resultados com a utilização de T1, sendo similar a T2 e significativamente superior aos demais tratamentos. Nos 
tratamentos sem adição do DMSO (T4 e T5), os resultados foram similares entre si (Tab. 1).

Para a VSL, apenas T3 foi significativamente inferior ao T1. A VAP também apresentou seus piores resultados em T3, sendo significativamente inferior aos demais tratamentos. Para VCL, T2 e T3 foram similares e significativamente inferiores aos outros tratamentos (Tab 1).

No que se refere à morfologia, também se verificou uma diminuição significativa de espermatozoides normais em todos os tratamentos quando comparados aos do sêmen fresco $(94,14 \%)$. Observando apenas os tratamentos, o T1 apresentou porcentagem de espermatozoides normais $(86,36 \%)$ significativamente superiores aos demais. Os tratamentos T2 $(79,71 \%)$ e $\quad$ T3 $(80,64 \%)$ apresentaram resultados semelhantes entre si e significativamente superiores aos tratamentos sem adição de DMSO (T4: 74,54\% e T5: $72,46 \%$ ), que, por sua vez, foram semelhantes entre si.

Foram encontradas correlações positivas entre VCL e taxa de espermatozoides morfologicamente normais $(r=0,415)$, VCL e VSL $(r=0,419)$, motilidade e VAP $(r=0,466)$, motilidade e VSL $(r=0,566)$, motilidade e taxa de espermatozoides morfologicamente normais $(\mathrm{r}$ $=0,687)$, VAP e VCL $(r=0,811)$ e VAP e VSL $(\mathrm{r}=0,825)$, todas com $\mathrm{P}<0,01$.

Apesar de a motilidade total, VSL e VAP terem sido semelhantes em T1 e T2, observou-se que o melhor tratamento foi o $\mathrm{T} 1$, pois, ao avaliar a VCL, T1 foi superior. Fisiologicamente é esperado que o espermatozoide de peixe tenha movimento circular, pois ele deverá percorrer uma trajetória curvilínea a fím de encontrar a micrópila para penetrar o ovócito. De fato, Viveiros et al. (2010) observaram que a VCL foi o principal parâmetro de velocidade que influenciou positivamente a capacidade fertilizante do sêmen pós-descongelação para Prochilodus lineatus.

Dessa forma, o presente trabalho enfatiza a importância de analisar, além da motilidade, as velocidades espermáticas como critério de avaliação da qualidade seminal pós- descongelação, auxiliando na elaboração de protocolos mais eficazes.

A principal correlação encontrada foi entre motilidade e taxa de espermatozoides morfologicamente normais, comprovando que, de maneira geral, uma boa estrutura espermática é necessária para se obter boas taxas de motilidade.

Apesar da ausência de crioprotetor interno nos tratamentos T4 e T5, foi observada a presença de espermatozoides móveis pós-descongelação, velocidades espermáticas semelhantes ao melhor tratamento (T1) e morfologia dos espermatozoides dentro dos padrões de normalidade exigidos para outros animais pelo Colégio Brasileiro de Reprodução Animal (2013; ainda não foi definido para sêmen de peixe). Esses resultados mostram uma ação protetora da associação ACP+AV.

Este é o primeiro trabalho que avalia o Aloe vera como componente do diluidor para criopreservação do sêmen de peixes. $\mathrm{Na}$ literatura é possível observar diversos modos de utilização do Aloe vera. Rodriguez et al. (1988) utilizaram o gel dessa planta para substituir o diluidor seminal na inseminação cervical e intrauterina de ovinos. Por conseguinte, em 2006, Gutiérrez et al. utilizaram o Aloe vera para congelação do sêmen ovino encontrando resultados satisfatórios de qualidade seminal. Em caprinos, Melo (2010a) utilizou o extrato bruto de Aloe vera em combinação com o $\mathrm{ACP}^{\circledR}$ para conservação de sêmen a $4^{\circ} \mathrm{C}$, e observou que essa combinação foi capaz de manter a qualidade espermática por até 48 horas.

A capacidade protetora da complexa composição $\mathrm{ACP}+\mathrm{AV}$ pode ocorrer devido à presença de inúmeras substâncias no Aloe vera, como polissacarídeos (glicose, galactose e xilose), tanino, esteroides, ácidos orgânicos, substâncias antibióticas, enzimas de vários tipos, resíduos de açúcar, uma proteína com 18 aminoácidos, vitaminas, minerais (sulfato, ferro, cálcio, cobre, sódio, potássio, manganês), dentre outros (Veloso e Peglow, 2003), e na ACP, como açúcares, gorduras neutras, vitaminas, minerais, aminoácidos (CQA, laudo $\mathrm{n}^{\circ}$ 96300/2009 e ITAL, laudo n CQ 4359/2009). 
Pode-se concluir que o uso do Aloe vera na forma de extrato bruto, como a utilizada neste trabalho, em associação ao DMSO na criopreservação de sêmen de tambaqui, não proporciona melhoria na proteção espermática durante o processo de criopreservação. No entanto, sua utilização em associação apenas com ACP, apesar da ausência de crioprotetor interno, permite a obtenção de taxa de morfologia espermática dentro dos padrões de normalidade e presença de espermatozoides móveis. Dessa forma, o uso do Aloe vera deve ser considerado em novos estudos, para avaliação da sua ação crioprotetora.

Palavras-chave: água de coco em Pó-104, crioprotetor, peixe, reprodução

\begin{abstract}
This study aimed to evaluate the extract of Aloe vera (AV) associated or not with $10 \%$ Dimethylsulfoxide (DMSO) in cryopreservation of tambaqui semen. For the formation of the pools $(n=14), 30$ males were hormonally induced twice. Each pool had the objective motility, curvilinear velocity, straight-line velocity, average path velocity and morphology analyzed before and after cryopreservation of semen. The means for cryopreservation were constituted of Powder Coconut Water-104 diluent added DMSO and/or AV (5 or 10\%). After cryopreservation, motility, velocities and morphology were reduced significantly when compared to fresh semen. For sperm motility the best treatment was that using only DMSO

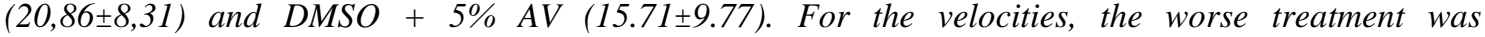
DMSO+10\% AV. Treatment with only the addition of DMSO had a significantly higher effect than others on percentage of morphologically normal sperm. The mean correlation found was between motility and the rate of morphologically normal sperm $(r=0.687)$. In conclusion, the addition of AV does not provide greater protection for spermatozoa during cryopreservation.
\end{abstract}

Keyword: Powder Coconut Water-104, cryoprotectant, fish, reproduction

\section{AGRADECIMENTOS}

Agradeço ao Departamento Nacional de Obras Contra as Secas (DNOCS) por ceder os animais e a estrutura para a realização do experimento; à empresa ACP Biotecnologia por fornecer o meio para criopreservação; à Fundação Cearense de Apoio ao Desenvolvimento Científico e Tecnológico (FUNCAP) pela concessão da bolsa de estudos; e à Financiadora de Estudos e Projetos (FINEP) pelo suporte financeiro para a realização dos experimentos.

\section{REFERÊNCIAS}

CARNEIRO, P.C.F.; AZEVEDO, H.C.; SANTOS, J.P.; MARIA, A.N. Cryopreservation of tambaqui (Colossoma macropomum) sêmen: extender, cryoprotectants, diluition ratios and freezing methods. Cryoletters, v.33, p.385-393, 2012.

GUTIÉRREZ, A.J.; COSME, R.W.; JIMÉNEZ, C.J.A.; RAMIREZ, G.J.A. Agua de coco, suero fetal bovino, Aloe vera y sus combinaciones para criopreservar semen ovino. Arch. Zootec., v.55, p.101-104, 2006.
LEITE, L.V.; OLIVEIRA, F.C.E.; NUNES, L.T. et al. Criopreservação do sêmen de tambaqui com ACP® adicionado de gema de ovo. Rev. Bras. Eng. Pesca., v.6, p.23-29, 2011.

MANUAL para exames andrológicos e avaliacão de sêmen animal. 3.ed. Belo Horizonte: CBRA, 2013.

MELO, C.C.S. Conservação de sêmen caprino a $4^{\circ} \mathrm{C}$ utilizando ACP-101® com duas concentrações de Aloe vera ou gema de ovo. 2010a. 72f. Dissertação (Mestrado em Ciências Veterinárias) - Programa de Pós-Graduação em Ciências Veterinárias, Universidade Estadual do Ceará, Fortaleza, CE.

MELO, M.A.P. Água de coco em pó (ACP-104) adicionada de crioprotetores sobre a morfometria da cabeça de espermatozoides de pirapitinga (Piaractus brachypomus) pósdescongelamento. 2010b. 63f. Dissertação (Mestrado em Ciências Veterinárias) - Programa de Pós-Graduação em Ciências Veterinárias, Universidade Estadual do Ceará, Fortaleza, CE. 
Aloe vera na criopreservação...

RODRIGUÉZ, F.; BALDASSARRE, H.; SIMONETTI, J. et al. Cervical versus intrauterine insemination of ewes using fresh or frozen semen diluted with Aloe vera gel. Theriogenology, v.30, p.843-854, 1988.

SALMITO-VANDERLEY， C.S.B.; VIEIRA, M.J.A.F.; LEITE, L.V. et al. Meios de congelação para conservação de sêmen de peixes da família Characidae. Cienc. Anim., v.22, nesp., p.255-268, 2012.

SREIT JR, D.P.; POVH, J.A.; FORNARI, D.C. et al. Recomendações técnicas para a reprodução do tambaqui. Teresina: Embrapa Meio Norte, 2012. 30p. (Documento, 212).
VELOSO, C.C.; PEGLOW, K. Plantas medicinais. Porto Alegre: EMATER/ ASCAR, 2003. 83p.

VIVEIROS, A.T.M.; NASCIMENTO, A.F.; ORFÃO, L.H.; ISAÚ, Z.A. Motility and fertility of the subtropical freshwater fish streaked prochilod (Prochilodus lineatus) sperm cryopreserved in powdered coconut water. Theriogenology, v.74, p.551-556, 2010. 